\title{
Intradermal nevocellular nevus with adenoid features: Report of a case
}

\author{
Tadashi Terada* \\ Department of Pathology, Shizuoka City Shimizu Hospital, Shizuoka, Japan
}

Received: January 3, 2016

DOI: $10.5430 /$ crcp.v4n1p26
Accepted: September 25, $2016 \quad$ Online Published: October 14, 2016

URL: http://dx.doi.org/10.5430/crcp.v4n1p26

\begin{abstract}
Intradermal nevocelluar nevus is a very common hamartomatous neoplasm of the skin. The author herein reports two tumors of intradermal nevocelluar nevus in the neck occurring in a 55-year-old woman. The tumors were characterized histologically by typical intradermal nevocelluar nevus with significant areas of adenoid or glandular formations. In both tumors, hair follicles were seen in centers of the tumors. The cellular features of the adenoid areas were the same as those of solid areas. In solid areas, vague vacuolated and obvious vacuolated cells were recognized in some areas. In solid areas of the surface and lateral areas of the tumors also showed clear cut adenoid features without vacuolations. The adenoid features showed mild depositions of hemosiderins. There were gradual merges between solid areas and adenoid or glandular areas. Histochemically, the tumor cells including adenoid structures had glycogen, but had no mucins. Immunohistochemically, cells of both tumors were strongly positive for S100 protein, vimentin, NSE, NCAM, and bcl-2, and were weakly positive for synaptophysin and chromogranin. The tumor cells showed low cell proliferative potential; the Ki-67 labeling index was $2 \%$. The tumor cells including adenoid structures were negative for CK AE1/3, CK CAM5.2, desmin, smooth muscle actin, HMB45, p53, p63, CD31, laminin, type 4 collagen, CEA. CD34, CA125, CA19-9, CD45, GFAP, KIT, PDGFRA, CD68, estrogen receptor, progesterone receptor, EMA, MUC1, MUC2, MUC5AC, and MUC6. These histological, histochemical, and immunohistocheimical findings strongly suggested that the present two tumors with adenoid structures belong to intradermal nevocelluar nevus. The formation of the adenoid architectures in the intradermal nevocelluar nevus was discussed. Possible mechanisms appear loss of cell adhesions molecules. The adenoid structures seem not to be degenerative ones. Pathologists should be aware that intradermal nevocelluar nevus may show adenoid structures to avoid diagnostic confusions, for example adenoma and adenocarcinoma of skin appendages. The occurrence of this hitherto undescribed condition in two intradermal nevocelluar nevus in a patient may imply genetic lineage. To elucidate the mechanisms of adenoid structures in intradermal nevocelluar nevus seems future work.
\end{abstract}

Key Words: Intradermal nevocellular nevus, Adenoid structures, Histopathology, Immunohistochemistry

\section{INTRODUCTION}

Nevocellular nevus $(\mathrm{NCN})$ or melanocytic nevus is a very common condition of melanocytic neoplasms which include, in addition to NCN, melanomas, blue nevus, combined lentigo, and NCN, nevus of Ito, Ohta, Spitz, Mongoloid, Clark, and Sutton and so on. NCN is generally occurs in young adults and increases in frequency in parallel with ageing, though it can occur in infant and early childhood. It can be excised by cosmetic reason and less frequently for differentiation from melanoma. NCN is histologically composed of nevoid melanocytes and classified into three types: junctional, intradermal, and compound. The nevocytes are

\footnotetext{
*Correspondence: Tadashi Terada, MD, PhD; Email: piyo0111jp@yahoo.co.jp; Address: Department of Pathology, Shizuoka City Shimizu Hospital, Miyakami 1231 Shimizu-Ku, Shizuoka 424-8636, Japan.
} 
located only in junctions in junctional $\mathrm{NCN}$, only in the dermis in intradermal NCN, and both in the junction and dermis in compound NCN. In general, the ontology of NCN shows the following scenario. The nervous system develops from ectoderm in embryo. It forms central and peripheral nervous systems. The nevocytes appear to develop from these nervous systems in fetal period and locates in the basal layer of the epidermis. The aberrant excess location of the nevocytes in epidermal basal layer is called junctional NCN. The nevocytes of junctional NCN fall into the dermis, thus finally creating compound $\mathrm{NCN}$. Thereafter, all the NC fall into dermis, creating intradermal NCN. Intradermal NCN, then, may show neuronal differentiation, and occasionally disappears in time from the skin. The morphology of the intradermal NCN is straightforward. In the upper position, round and giant melanocytes with melanin pigmentation are seen. The nevocytes forms solid nests in middle area, and neural differentiation is frequently seen in deep areas. The intradermal NCN is a well-defined, symmetrical lesion without invasive features. Malignant transformation of $\mathrm{NCN}$ is exceptional.

The intradermal NCN showed solid nests of NC. The author recently encountered an intradermal NCN with marked adenoid changes. A review of the literatures and text books revealed no such a case. Herein reported is a case of such an intradermal adenoid NCC with immunohistochemical studies. Such a case has not been reported, to the best of the author's knowledge. Thus, the present case may represent the first hitherto undescribed variant (intradermal adenoid $\mathrm{NCN}$ ) of NCN.

\section{CASE REPORT}

A 55-year-old woman consulted our hospital because of two black tumors in the neck. Physical examination revealed two polypoid tumors of the neck; one was $11 \mathrm{~mm} \times 12 \mathrm{~mm}$ $\times 10 \mathrm{~mm}$ and another was $9 \mathrm{~mm} \times 10 \mathrm{~mm} \times 9 \mathrm{~mm}$. The gross features of the two lesions were typical for intradermal NCN. Resection of the tumors was performed with the use of anesthetic solution.

Histologically, the two tumors were histologically identical tumors (see Figure 1A). One tumor had apparent hair follicle in the center of the tumor (see Figure 1A) and another had possible hair follicles in the tumor center. Both tumors were composed of solid areas and adenoid or glandular areas (see Figure 1A-D). There were frequent transitions between solid and adenoid areas in the NCN (see Figure 1A). The stroma adjacent to the tumor showed focal mucinosis (see Figure 1AC). The superficial part showed hyperpigmentation and giant cells (see Figure 1A-B); the presence of melanin confirms that the tumor are melanocytic ones. The lower parts showed

Published by Sciedu Press vague neuroid differentiation (see Figure 1A). The adenoid areas showed well developed adenoid or tubular structures (see Figure 1B-C). No apparent mucins were seen in the lumens. Mild deposition of hemosiderin positive for Prussian blue reaction was seen (see Figure 1B-C). No apparent features of epithelial cells were seen. The non-adenoid solid area shows ordinary NCN or neurofibromatous features (see Figure 1D). No atypical features were seen in the solid areas (see Figure 1D). However, in the solid area, there were mild vacuolar changes of the tumor cells (see Figure 1D), and apparent vacuolation was seen in the solid areas. The outer parts of the surface areas frequently showed adenoid features of single cells or clustered cells (see Figure 1A). Individual cell morphology of the adenoid areas and solid areas was the same (see Figure 1A-D). In total, no atypical features were seen. No epidermotropisms or deep invasions were seen (see Figure 1A). The features were typical for intradermal NCN except for the adenoid or glandular features. The adenoid structures were not similar to vascular structure, being devoid of endothelial cells, pericytes, basement membrane, and smooth muscle cells.

A histochemical study was performed, as described previously. ${ }^{[1,2]}$ Mucicarmine and colloidal iron stains were negative. Periodic acid-Schiff (PAS) stain showed a little glycogen. PAS after diastase digestion (d-PAS) and alcian blue (AB) at $\mathrm{pH} 2.5$ and $\mathrm{pH} 1.0$, and combined d-PAS/AB pH2.5 and $\mathrm{pH} 1.0$ showed no mucins. The brown pigments were positive with Masson-Fontana stain, being melanin. Some brown pigments seen in the adenoid area were positive with Prussian blue technique.

An immunohistochemical study was performed with the use of Dako Envision technique (Dako Corp, Glostrup, Denmark) as described previously. ${ }^{[3,4]}$ Immunohistochemically, the solid and adenoid areas showed the same immunohistochemical patterns. The tumor cells were strongly positive for S100 protein (see Figure 2A), vimentin (see Figure 2B), neuron-specific enolase (NSE) (see Figure 2C), NCAM (see Figure 2D), and bcl-2. They were weakly positive for synaptophysin (see Figure 2E) and chromogranin (see Figure 2F). The Ki-67 labeling index was $2 \%$. They were negative for cytokeratin (CK) AE1/3, CK CAM5.2, desmin, smooth muscle actin, HMB45, p53, p63, CEA, CD31, CD34, laminin, type 4 collagen, CA125, CA19-9, CD45, GFAP, KIT, PDGFRA, CD68, estrogen receptor (ER), progesterone receptor (PGR), EMA, and MUC1, MUC2, MUC5AC, and MUC6. Neither endothelial antigens (CD31 and CD34) nor basement membrane antigens (laminin and type 4 collagens) were recognized.

In both tumors, the morphological features were those of 
typical intradermal NCN except for adenoid formations. The immunohistochemical features were also typical features of intradermal NCN except for adenoid features. Therefore, two intradermal adenoid NCNs were diagnosed by the author.
The patient was followed up, and no recurrence or metastasis was seen after 2 years of the resection. The patient is now healthy.
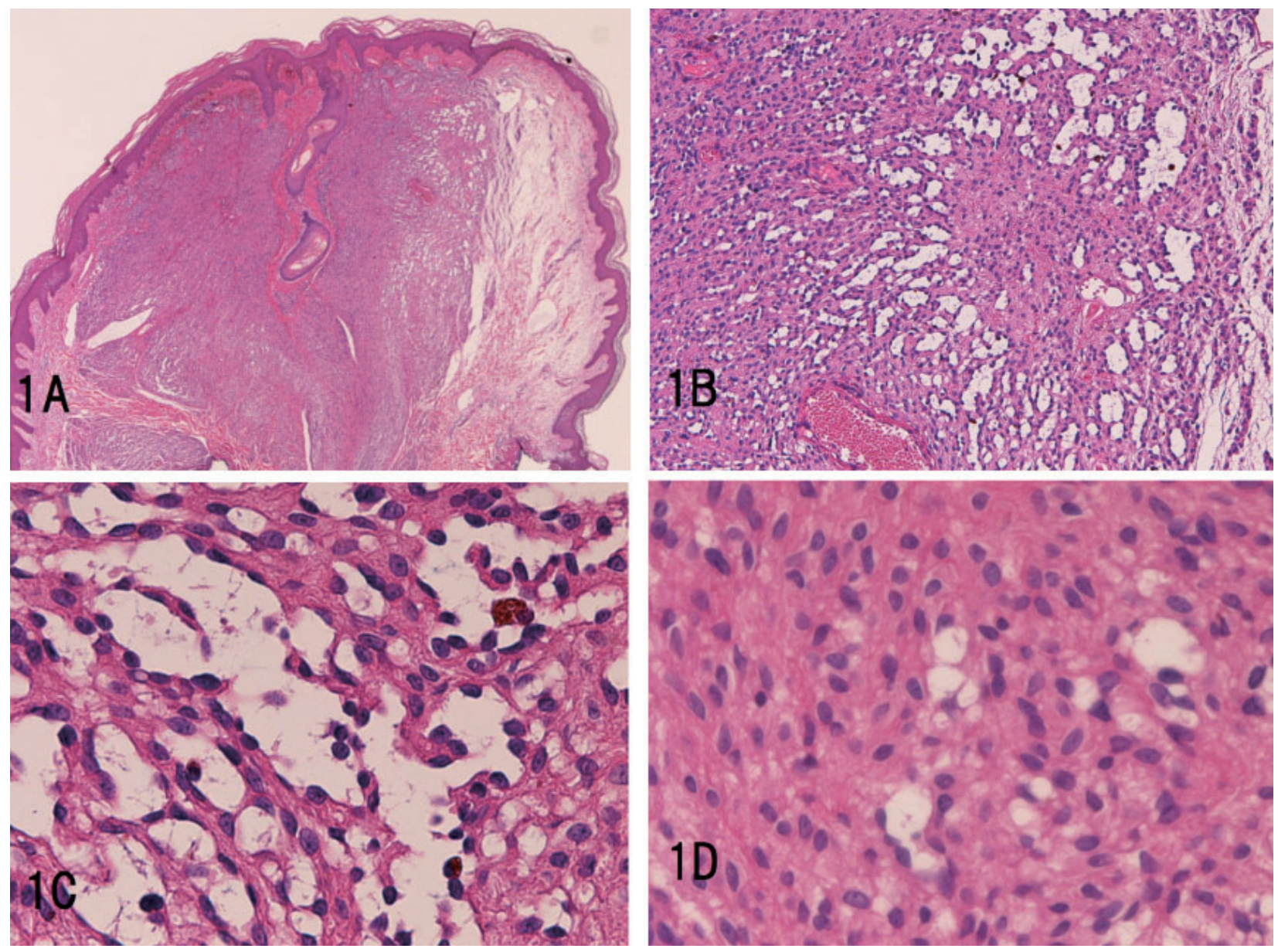

Figure 1. Histology of the two intradermal nevocellular nevi with adenoid features. Some of the lumens of the adenoid features are marked by asterisks.

A: One tumor showing typical features of intradermal nevocellular nevus except for adenoid structures. A hair follicle is seen in the intradermal nevocellular nevus HE, $\times 20$. B: Medium size view. The adenoid architectuires within the intradermal nevocellular nevus are apparent. HE $\times 100$. C: High power view of the adenoid areas. The adenoid structures are obvious. A few hemosiderin pigments are seen. HE, $\times 250$. D: Solid area. High power view. The features are ordinary intradermal nevocellular nevus. Apparently vacuolated cells are seen. $H E, \times 250$.

\section{Discussion}

The histological features of the present two tumors are typical intradermal NCN except for the adenoid features. An extensive review of the literature and text book was performed. However, the author could not find such NCN with adenoid features. Thus these two tumors were hitherto undescribed entity of intradermal NCN, to the best of the author's knowledge. However, a few recent studies showed the presence of vascular-like structures in the NCN. ${ }^{[5,6]}$ Review of their cases suggests a resemblance of the present cases to those of them. The present case can be included in such cases of NCN with vascular-like structures. However, the intercellular lumens in the present case did not resemble vascular lumens but were similar to glandular structures, the lumens being free from red blood cells, leukocytes and lymph fluids. It is interesting that the structures with lumens were negative for basement lumen antigens (laminin and type 4 collagens) and endothelial markers (CD31 and CD34); these were reported in vascular-like structures in NCN. ${ }^{[5,6]}$

The cell type of the adenoid areas was the same as that of solid areas in both tumors. Thus, the adenoid areas are usual 
tumor constituent of NCN except for the peculiar structures. In one tumor, the central area contained a hair follicle and the other tumor a probable hair follicle. These findings suggest that the adenoid formation is related to the hair structures.
However, the adenoid areas showed no features of hair follicles, sebaceous glands, or sweat glands.
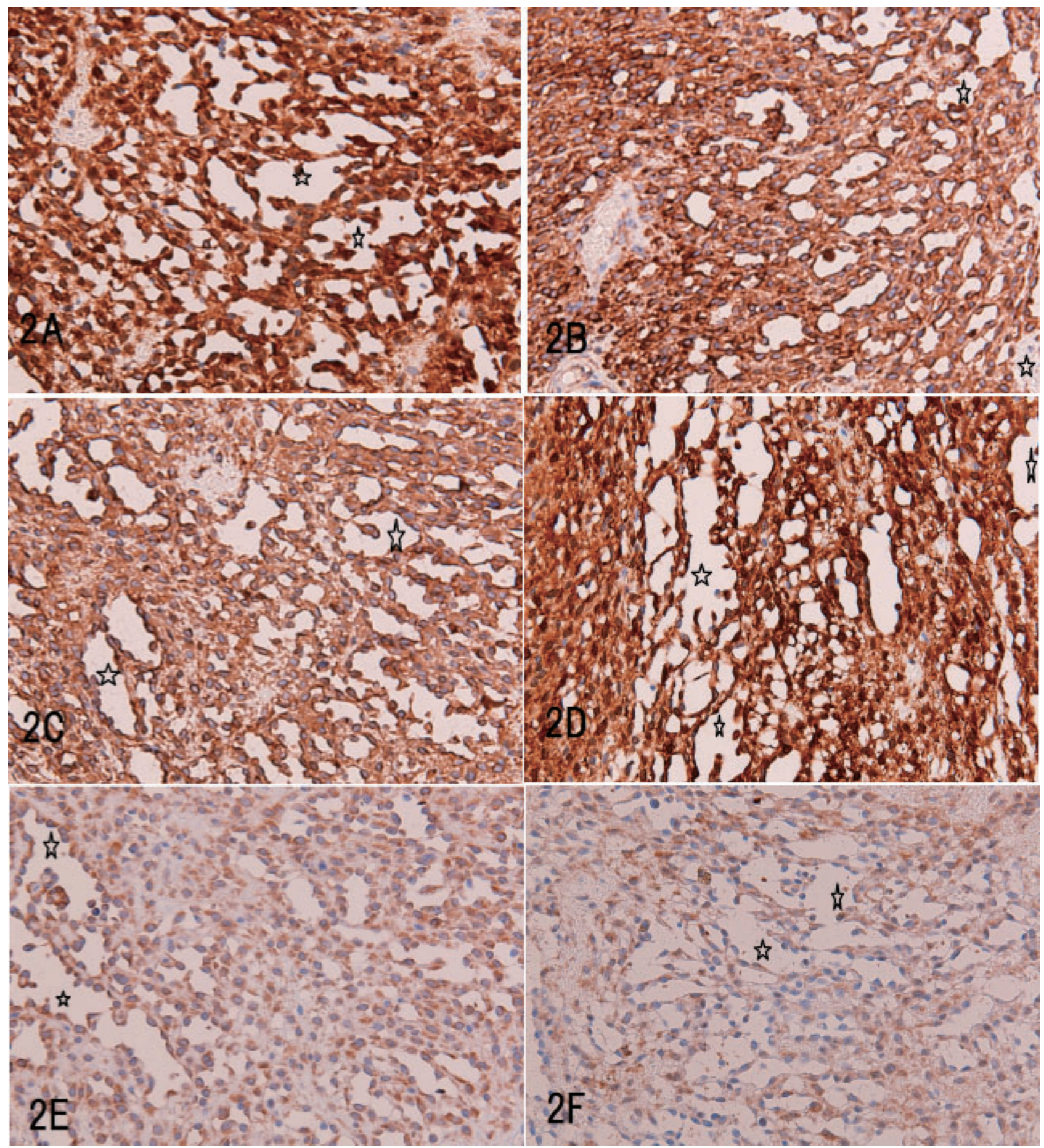

Figure 2. Immunohistochemical findings of the intradermal nevocellular nevus with adenoid features

Some of the lumens of the adenoid features are marked by asterisks. The tumor cells were strongly positive for S100 protein (A), vimentin $(B)$, neuron-specific enolase $(C)$, and NCAM $(D)$. . They were weakly positive for synaptophysin $(E)$ and chromogranin $(F)$.

Immunostaining: A-F; $\times 200$. 
The adenoid areas show a few hemosiderin pigments, suggesting previous minute hemorrhage in the adenoid areas. However, there were apparent adenoid formations in the solid areas. In contrast, the both tumors had areas of vague vacuolar changes of the nevocytes. These vacuolar changes were intermediate between the adenoid features and vacuolar changes. The author thinks that the adenoid changes are at least in part associated with the vacuolar changes seen in the solid areas. The apparent adenoid features were seen abruptly in solid areas in the peripheral surface areas of the NCN. These findings may suggest that the adenoid changes are coherent alterations of these NCN.

The histochemistry showed a few glycogens in the NCN cells, suggesting that the NCN cells have glycogens and use them as energy. The histochemistry did not show any mucins. These findings highly suggest the adenoid areas and vacuolated cells in solid areas are not due to accumulation of mucins. This also suggests that the adenoid and vacuolated cells are not degenerative cells. The finding also strongly suggests that the adenoid and vaculated cells are not related to the characteristics of mucin forming adenonomas or adenocarcinomas. ${ }^{[7-9]}$ However, it is possible that the adenoid changes of the present $\mathrm{NCN}$ are among artefact caused, for example, by anesthetic solution used during the biopsy.

Immunohistochemically, the solid and adenoid areas showed the same immunohistochemical patterns, suggesting that both areas are essentially the same. The tumor cells were strongly positive for S100 protein, vimentin, NSE, NCAM, and bcl-2, and were weakly positive for synaptophysin and chromogranin. In melanocytic tumors, histochemistry of Masson-Fontana detecting melanin pigment and immunohistochemistry for S100 protein, HMB45, and Melan A were powerful tools. The present study did not perform Melan A because hepatoxylin and eosin (HE) histology was typical for intradermal NCN. In the present tumor, the tumor cells were strongly positive for $\mathrm{S} 100$ protein but negative for HBD45, a melanosome antigen. The negativity of HMB45 in the present tumors are relatively atypical, because most NCN are positive for HMB45 though the positivity is mild or slight. In the present tumor, neuroendocrine antigens including NCAM, NSE, chromogranin and synaptophysin were strongly or weakly positive, suggesting that the present tumor is intradermal NCN. In the present tumors, vimentin was strongly positive but CK AE1/3 and CK CAM5.2 were negative, suggesting that the present tumors are mesenchymal tumors and are compatible with the diagnosis of intradermal NCN. The present tumor showed positive Ki-67 antigen (2\%), demonstrating that the involuting intradermal NCN has as yet cell proliferative activity. However, the Ki67 labeling index was low and $2 \%$, suggesting cell proliferation is limited in intradermal NCN. In the present tumor, desmin, smooth muscle actin was negative, implying that the present tumor is not smooth muscle or vascular tumors. The present tumor was negative for p53, suggesting the benign nature of the present tumor. The present tumors were negative for CEA, CA125, and CA19-9, suggesting that the adenoid areas of the tumors are not glandular structures. The present tumor was negative for p63, EMA, and GFAP, suggesting that the present tumors are not epithelial or myoepithelial tumors. The present tumor is negative for CD34, suggesting that the tumors are not CD34-related tumor such as solitary fibrous tumors. In addition, the negativity of the present tumor for endothelial antigens (CD31 and CD34) and basement membrane antigens (laminin and type 4 collagens) indicates that the present tumor is not vascular or lymphocytic neoplasms. The present tumor is negative for CD45, suggesting that the tumors are not white blood cell tumors such as lymphomas. The present tumors were negative for ER and PgR, suggesting that the present tumors are not hormone-related tumors. The present tumors are negative for MUC apomucins, suggesting again that the adenoid areas are not glandular lumens. The MUC profiles are compatible with the mucin histochemistry. Although the tumor cells were negative for CD68, CD68-positive macrophages were scattered in the tumors. It was a little out of anticipation that the present tumor was negative for KIT and PDGFRA, which are frequently positive for neuroendocrine tumors and melanomas; however, it is not strange. The tumor was strongly positive for bcl-2 tyrosine kinase oncoprotein. Bcl-2 is an apoptosis inhibitor and label follicular lymphoma and stem cells. The positive reaction of bcl-2 in NCN and melanomas has been reported. It may be expressed in $\mathrm{NCN}$ and more often in malignant melanoma and is a useful marker of melanoma. ${ }^{[10-12]}$

The reason for the adenoid structures in the present intradermal NCN is only speculative. Cell adhesion molecules such as E-cadherin-catenins systems may be present in NCN. Tonofilaments and desmozomes adhesion may be present in NCN. These molecules have not been studied in NCN. The present study also did not investigat the expressions of cell adhesion molecules. However, the author thinks that these cell adhesion molecules may be destroyed in the current tumors and gave rise to the adenoid patterns in NCN. Similar situations are seen in cutaneous acantholytic squamous cell carcinoma in which desmosomal proteins are destroyed. This hypothesis must be elucidated in future.

In practical sense, the adenoid structures in intradermal NCN may be misinterpreted as adenocarcinoma or other tumors forming adenoid pattern such as sweat glands adenoma and adenocarcinoma, sebecerous adenoma, folliclar neoplasms with adenoid differentiations, particularly very small biopsy 
samples. Thus, pathologists should know that the intradermal NCN may show adenoid features. Finally, in the present case, both two tumors of NCN showed adenoid features. This may suggest a genetic predisposition of such a tumor.

\section{Conclusions}

The author presented two peculiar NCN with adenoid features. The present case can be similar to a few rare variants of NCN previously reported as NCN with vascular-like struc- tures. Although the mechanism for adenoid formation in intradermal NCN could not be explained, pathologist should know that NCN can show adenoid features to avoid pathologic diagnostic confusions, for example adenoma and adenocarcinoma of skin appendages. To solve the mechanisms of the formation of adenoid features within $\mathrm{NCN}$ are fascinating works of the future.

\section{CONFlicts of InTEREST Disclosure}

The author has no conflict of interest.

\section{REFERENCES}

[1] Terada T, Nakanuma Y, Ohta G. Glandular elements around the intrahepatic bile ducts in man; their morphology and distribution in normal livers. Liver. 1987; 7(1): 1-8. PMid: 3553822. http: //dx.doi.org/10.1111/j.1600-0676.1987.tb00308.x

[2] Terada T, Nakanuma Y. Morphological examination of intrahepatic bile ducts in hepatolithiasis. Virchows Arch A Pathol Anat Histopathol. 1988; 413: 167-76. PMid: 3133876. http://dx.d oi.org/10.1007/BF00749679

[3] Terada T, Kawaguchi M, Furukawa K, et al. Minute mixed ductalendocrine carcinoma of the pancreas with predominant intraductal growth. Pathol Int. 2002; 52: 740-6. PMid: 12685552. http: //dx.doi.org/10.1046/j.1440-1827.2002.01416.x

[4] Terada T, Kawaguchi M. Primary clear cell adenocarcinoma of the peritoneum. Tohoku J Exp Med. 2005; 271: 271-5. http: $/ / \mathrm{dx}$.doi.org/10.1620/tjem.206.271

[5] Collina G, Eusebi V. Naevocytic naevi with vascular-like spaces. Br J Dermatol. 1991; 124: 591-5. PMid: 2064945. http://dx. doi.o $\mathrm{rg} / 10.1111 / \mathrm{j} .1365-2133.1991 . t b 04957 . \mathrm{x}$

[6] Demitsu T, Kakurai M, Yamada T, et al. The vascular space-like structure in melanocytic nevus is not an injection artifact: report of a case and an experimental study. J Dermatol. 1998; 25: 143-9. PMid: 9575674. http://dx.doi.org/10.1111/j.1346-8138. 1998.tb02369.x
[7] Terada T, Nakanuma Y. Development of human intrahepatic peribiliary glands: Histological, keratin immunohistochemical and mucus histochemical analyses. Lab Invest. 1993; 68: 261-9. PMid: 7680729.

[8] Terada T, Nakanuma Y. Profiles of expression of carbohydrate chain structures during human intrahepatic bile duct development and maturation: a lectin-histochemical and immunohistochemical study. Hepatology 1994; 20: 388-97. PMid: 8045500. http: //dx.doi.org/10.1002/hep.1840200219

[9] Terada T. Small cell neuroendocrine carcinoma of the prostate: Incidence and a report of four cases with an examination of KIT and PDGFRA. Prostate. 2012; 72: 1150-6. PMid: 22127977. http: //dx.doi.org/10.1002/pros. 22464

[10] Terada T. Primary small cell carcinoma of the ureter: a case report with immunohistochemical and molecular genetic analysis of KIT and PDGFRA genes. Pathology. 2010; 42: 101-2. PMid: 20025496. http://dx.doi.org/10.3109/00313020903443018

[11] Terada T. Autopsy case of primary small cell carcinoma of the urinary bladder: KIT and PDGFRA expression and mutations. Pathol Int 2009; 59: 247-50. PMid: 19351368. http://dx.doi.org/10.11 $11 / j \cdot 1440-1827.2009 .02358 \cdot x$

[12] Terada T. Primary esophageal small cell carcinoma with brain metastasis and with CD56, KIT, and PDGFRA expressons. Pathol Oncol Res. 2012; 18: 1091-3. PMid: 21626441. http://dx.doi.org/1 $0.1007 / \mathrm{s} 12253-011-9374-\mathrm{y}$ 